\title{
Long-Term Stability Studies for the Large Hadron Collider
}

\author{
F. Galluccio, Z. Guo, W. Scandale, F. Schmidt and A. Verdier \\ CERN \\ CH-1211 Geneva 23
}

\begin{abstract}
The dynamic aperture of the next generation of hadron colliders will be strongly limited by the non-linear components of the bending and focusing fields, which are unavoidable in superconducting magnets. In the Large Hadron Collider (LHC) particle stability over about $10 \mathrm{~min}$ is required at injection: this corresponds to $7 \times 10^{6}$ turns in the machine. Tracking simulations up to such a high number of turns for a significant number of different cases is impractical, if not impossible, even with the most powerful computer; therefore a different approach must be used to evaluate the LHC long-term dynamic aperture. The most realistic machine has been modelled, including RF cavities, residual closed-orbit distortion after compensation, systematic and random multipolar errors in dipoles and quadrupoles, skcw quadrupoles, and lumped multipolar correctors. Long-term computer tracking $\left(4 \times 10^{5}\right.$ turns/ $\sim 10 \mathrm{~s}$ ) has been performed for a large number of machine configurations, for particles with different starting coordinates and momenta. The horder between stable and chaotic motion has been estimated from the analysis of the medium term tracking results using the Lyapunov exponent method. Only for a small number of selected cases has tracking been resumed and carried on up to $10^{6}$ turns. The results of this study and the estimated value of the long-term dynamic aperture of the LHC at injection are given here.
\end{abstract}

\section{Introduction}

No analytical tool is yet available to allow a fast and reliable prediction of the long-term dynamic aperture for conservative systems like the Large Hadron Collider. The reason for this is twofold: first, it is difficult to detect an instability that is caused by the interaction of many weak resonances, and secondly, finding this border of stability, for instance via the Lyapunov method, does not tell us when the actual particle loss will take place. It therefore remains mandatory to do tracking over many turns to estimate the range of amplitudes for which the storage and the acceleration of particles can be ensured. It goes without saying that analytical tools are of paramount importance to understand the results.

In order to make use of the power of today's computers such as the Cray-XMP at CERN, the tracking codes MAD [1] and SIXTRACK [2] have been vectorized. The use of two independent programs, having about the same

*On leave of absence from IHEP, Beijing, China. computational speed $\left(2 \times 1 U^{\circ} \mathrm{s}\right.$ of $\mathrm{CP} U$ tor $2 U$ particies Iollowed over $10^{6}$ turns), allowed these costly investigations to be double-checked. Most of the tracking data have been recorded on tape, to allow further analysis when new aspects arise.

The effect of rounding errors is always a matter of concern when up to $10^{12}$ operations are performed for $10^{6}$ turns of a full LHC structure. It has been checked that with single precision on the Cray these errors do not invalidate our results up to $10^{6}$ turns.

We therefore feel confident that our tracking studies allow, in the limit of the physical model, a safe estimate of the LHC dynamic aperture.

\section{The Machine Model}

The layout and the optics of the LHC as used for these studies are described in Ref. [3]. A thin-lens lattice model is adopted for tracking, which has been shown to give results nearly identical to those of the thick-lens model. The machine consists of eight arcs and eight straight sections, with general-purpose insertions in straight sections 4,6 , and 8 , experimental insertions in straight sections 1,2 , and 5 , a dump insertion in straight section 3 and a halocleaning insertion in straight section 7 . At injection all general-purpose and experimental insertions are detuned with $\beta_{x}^{*}=\beta_{y}^{*}=8 \mathrm{~m}$; in the dump insertion $\beta_{x}^{*}=\beta_{y}^{*}=$ $220 \mathrm{~m}$, and in the cleaning insertion $\beta_{x}^{*}=\beta_{y}^{*}=15 \mathrm{~m}$.

' ' 'he selected tunes are $\mathrm{Q}_{x}=70.28$ and $\mathrm{Q}_{y}=70.31$; this working point lies inside one of the three best regions of tunes (Fig. 1) and at a distance to the diagonal that minimizes the effects of coupling [4].

The performances of a superconducting machine like the LHC are determined by the multipolar imperfections of the magnetic fields, and by the correction elements introduced to reduce their influence. In order to simulate the machine in the most realistic way, all linear and non-lincar perturbations and their respective correction elements are implemented in the model.

The systematic and random multipolar errors of all dipoles and quadrupoles [5], with both normal and skew components, and up to the 20-pole, can be found in Table 1. Moreover, a closed-orbit distortion is simulated by misaligning all quadrupoles.

We have included the following correction elements: four families of skew quadrupoles are located in the insertions to compensate linear coupling, orbit correction dipoles are 


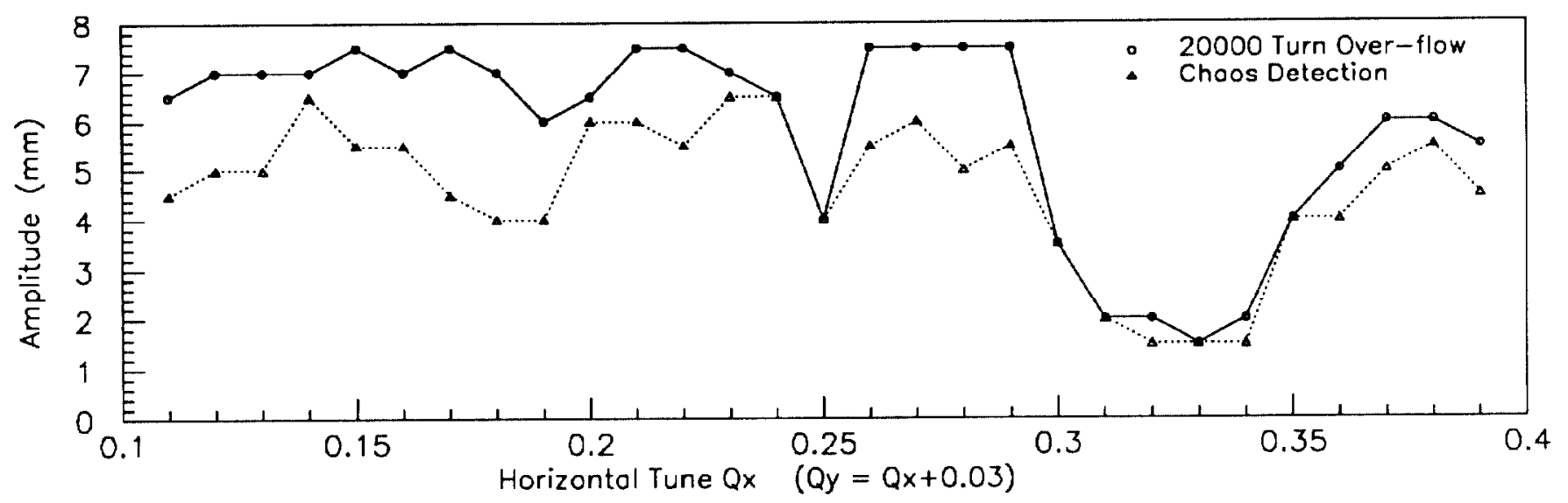

Figure 1: Dynamic Aperture as a Function of the Working Point

Table 1: Coefficients of Systematic and Random (r.m.s.) Multipolar Errors (in units of $10^{-4}$ at $\left.R_{r}=1 \mathrm{~cm}\right)$ at Injection

\begin{tabular}{|c|ll|ll|ll|l|l|}
\hline \multirow{2}{*}{ Order } & \multicolumn{4}{|c|}{ Dipole Errors } & \multicolumn{3}{c|}{ Quadrupole Errors } \\
\cline { 2 - 9 } & \multicolumn{2}{|c|}{ Systematic } & \multicolumn{2}{|c|}{ Random } & \multicolumn{2}{c|}{ Systematic } & \multicolumn{2}{c|}{ Random } \\
& $b_{n}$ & $a_{n}$ & $b_{n}$ & $a_{n}$ & $b_{n}$ & $a_{n}$ & $b_{n}$ & $a_{n}$ \\
\hline 1 & 0.0 & 0.0 & 0.0 & 0.0 & 0.0 & 0.0 & 0.0 & 0.0 \\
2 & 3.00 & 0.80 & 1.2 & 1.7 & 0.0 & 0.0 & 0.0 & 0.0 \\
3 & -3.35 & 0.10 & 1.7 & 0.5 & 0.02 & 0.28 & 1.06 & 1.04 \\
4 & 0.05 & 0.03 & 0.15 & 0.2 & 0.19 & 0.01 & 0.24 & 0.39 \\
5 & 0.45 & 0.03 & 0.22 & 0.07 & 0.05 & 0.01 & 0.10 & 0.09 \\
6 & 0.0 & 0.0 & 0.0 & 0.0 & -0.43 & 0.0 & 0.19 & 0.07 \\
7 & 0.14 & 0.02 & 0.02 & 0.04 & 0.0 & 0.0 & 0.02 & 0.03 \\
8 & 0.0 & 0.0 & 0.0 & 0.0 & 0.0 & 0.0 & 0.01 & 0.01 \\
9 & 0.034 & 0.001 & 0.005 & 0.002 & 0.0 & 0.0 & 0.01 & 0.01 \\
10 & 0.0 & 0.0 & 0.0 & 0.0 & 0.01 & 0.0 & 0.01 & 0.0 \\
\hline
\end{tabular}

available next to each quadrupole, and lumped multipolar correctors, placed 'à la Neuffer', are included in every cell in the arcs.

The linear coupling compensation and the closed orbit correction are extensively reviewed in these proceedings [4]. The compensation of the amplitude- and momentum-dependent detuning due to the systematic multipolar errors of the dipoles is accomplished by means of multipole correctors (sextupoles, octupoles, and decapoles). Their strengths are set by minimizing the chromaticity up to third order [6], which also lcads to a strong reduction of the amplitude-dependent tune-shift.

Extensive studies have shown that the multipolar corrector in the center of the half-cell is mandatory, except, maybe, for the octupole.

\section{Long-Term Tracking}

The many parameters that may limit the stability of the LHC make it difficult to provide a reliable estimate of the dynamic aperture. Our first task was therefore to find a small but sufficient set of those parameters to reduce the enormous need of computing time, thereby allowing a repetition of the whole study in case of changes during the design period.

The synchrotron motion is routinely included since tracking with fixed momentum deviation has shown to be too optimistic. We consider transversely round beams and we fix the relative momentum deviation to $10^{-3}\left(2 \sigma_{E}\right.$ at injection). Moreover, we can post-process those data to obtain detuning, smear and other quantities of interest. An early detection of chaos via the Lyapunov exponent method provides us with an estimate of the border of infinite stability and the actual particle loss is recorded in survival plots for up to $4 \times 10^{5}$ or even $10^{6}$ turns. A critical issue is how to assign the random errors to each individual element. From 10 sets of random distributions the worst, best, and most realistic ones were selected on the basis of short-term tracking (1000 turns). In order not to mix different effects, this choice of random distributions was done separately for dipoles, quadrupoles and quadrupole alignment errors.

\section{Results}

Figure 2 shows a typical example of a survival plot, in this case with multipolar errors in the dipoles only. The tracking has been carried on up to $10^{6}$ turns. For the three selected random error distributions we find a steep increase of the loss turn number (above $10^{5}$ ) when the Lyapunov limit is approached. The unexpectedly large dispersion of the dynamic aperture obtained for different random distributions remains to be understood. It is hoped, however, that it can be reduced by sorting the magnets.

Table 2 gives a summary of the results of our study for the most realistic error distribution: in all cases the border between regular and chaotic particle amplitudes is given, together with the largest stable amplitude for the indicated number of turns.

The rather large $b_{7}$ and $b_{9}$ field components lead to a reduction of the dynamic aperture by $1.5 \mathrm{~mm}$ (cases Nos. 1 and 2) in the presence of dipole errors only. Adding 


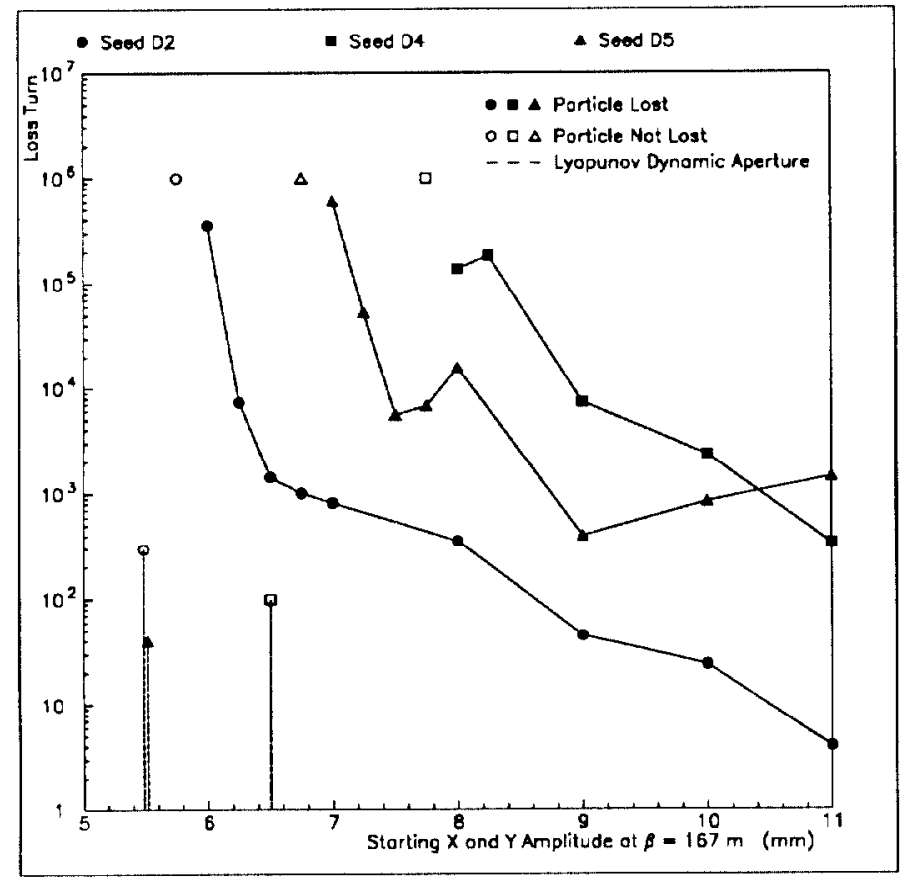

Figure 2: Survival Plot for the LHC with Errors in the Dipoles Only - 3 Random Error Distributions

the quadrupole errors does not change the dynamic aperture in the limit of our resolution of $0.25 \mathrm{~mm}$. The skew quadrupole components $\left(a_{2}\right)$ in the dipoles, notably the large random part, also lead to a significant decrease of the dynamic aperture of $0.75 \mathrm{~mm}$ (case No. 4). A small closed orbit (case No. 5) does not affect the stability behaviour very much, whilst a sizeable and probably realistic orbit of $\sigma=1.7 \mathrm{~mm}$ causes an additional drop of more than $1 \mathrm{~mm}$ (case No. 6). For a safe operation of the LHC it is believed that an aperture of $4.5 \sigma$ of the beam is needed. In our case this corresponds to $5.2 \mathrm{~mm}$, which is larger than our overall dynamic aperture of $3.75 \mathrm{~mm}$.

Increasing the dipole bore diameter from 50 to $56 \mathrm{~mm}$, a large fraction of the loss in the dynamic aperture has been recovered (cases Nos. 2 and 7), so that the required stable region is achievable. A similar improvement of the dynamic aperture has been obtained by reducing the $b_{7}$ and $b_{9}$ components by a factor of 2 and 3 , respectively. Another amclioration can be expected from the reduction of the random $a_{2}$ component. It remains to be seen which strategy for increasing the dynamic aperture is followed.

Although the performance of the machine at injection is believed to be more critical, we still have to investigate the behaviour of the machine in collision mode.

\section{Acknowledgements}

We are very grateful to $\mathrm{E}$. McIntosh from the CN Division of CERN who assisted us in all questions concerning computing, and allowed us to make the best use of our facilities, including data transfer between computers and data convertibility between programs, graphics and
Table 2: Dynamic Aperture in $\mathrm{mm}$ at a Focusing Quadrupole $(\beta=167 \mathrm{~m})$

\begin{tabular}{|l|c|c|c|}
\hline \multirow{2}{*}{ Machine Configuration } & \multicolumn{3}{|c|}{ Dynamic Aperture based on: } \\
\cline { 2 - 4 } & $\begin{array}{c}\text { Chaos } \\
\text { Detection }\end{array}$ & $\begin{array}{c}10^{5} \text { turn } \\
\text { overflow }\end{array}$ & $\begin{array}{c}4 \times 10^{5} \text { turn } \\
\text { overflow }\end{array}$ \\
\hline $\begin{array}{l}\text { 1) Dipole }(=50 \mathrm{~mm}) \text { Err. } \\
\text { No } a_{2}-\text { No Syst. } b_{7}, b_{9}\end{array}$ & 7. & 9. & \\
\hline $\begin{array}{l}\text { 2) Dipole }(=50 \mathrm{~mm}) \text { Err. } \\
\text { No } a_{2}-\text { With Syst. } b_{7}, b_{9}\end{array}$ & 5.50 & 7.00 & 7.00 \\
\hline $\begin{array}{l}\text { 3) Dipole }(=50 \mathrm{~mm}) \text { and } \\
\text { Quad. }(\phi=56 \mathrm{~mm}) \text { Err. } \\
\text { No } a_{2}-\text { With Syst. b7, } b_{9}\end{array}$ & 5.75 & 7.25 & 6.75 \\
\hline $\begin{array}{l}4) \text { Dipole }(=50 \mathrm{~mm}) \text { and } \\
\text { Quad. }(\phi=56 \mathrm{~mm}) \text { Err. } \\
\text { All Multipole Errors }\end{array}$ & 5.0 & 6.5 & 6.0 \\
\hline $\begin{array}{l}5) \text { All Multipole Errors in } \\
\text { Dipoles and Quads. } \\
+ \text { Orbit }(\sigma=0.6 \mathrm{~mm})\end{array}$ & 4.75 & 6.75 & 6.50 \\
\hline $\begin{array}{l}6) \text { All Multipole Errors in } \\
\text { Dipoles and Quads. } \\
+ \text { Orbit }(\sigma=1.7 \mathrm{~mm})\end{array}$ & 3.75 & 5.75 & 5.25 \\
\hline $\begin{array}{l}\text { 7) Dipole }(\phi=56 \mathrm{~mm}) \text { and } \\
\text { Quad. }(=56 \mathrm{~mm}) \text { Err. } \\
\text { No } a_{2}-\text { With Syst. } b_{7}, b_{9}\end{array}$ & 6.25 & 8.25 & 8.25 \\
\hline
\end{tabular}

the like. F. Zimmermann from DESY helped us modify SIXTRACK to allow the analysis of the correction scheme. We finally would like to thank $\mathbf{J}$. Gareyte for many stimulating discussions and continuous support.

\section{References}

[1] H. Grote and F. C. Iselin, "The MAD Program (Methodical Accelcrator Design) Version 8.1, User's Reference Manual", CERN-SL 90-13 (AP).

F. C. Iselin, "Improvements in MAD in View of LHC Design", These Proceedings.

[2] F. Schmidt, "SIXTRACK Version 1, Single Particle Tracking Code Treating Transverse Motion with Synchrotron Oscillations in a Symplectic Manner, User's Reference Manual", CERN-SL 90-11 (AP).

F. Schmidt and $M$. Vänttinen, "Vectorization of the Single Particle Tracking Program SIXTRACK", CERN SL/AP/Note/90-20.

[3] W. Scandale, "The Lattice of the LHC: Version 1", CERN-SL 91-03 (AP), LHC Note 139.

[4] F. Galluccio, Z. Guo, T. Risselada, W. Scandale and F. Schmidt, "Compensation of Linear Lattice Imperfections in the Large Hadron Collider", These Proceedings.

[5] The LHC Study Group, "Design Study of the Large Hadron Collider (LHC)", CERN 91-03, May 1991.

[6] F. Galluccio, "Compensation of Systematic Multipolar Errors in the LHC from the Operational Point of View", CERN SL/AP/Note/90-12, LHC Note 133. 